\title{
High redshift quasars monitoring campaign
}

\author{
Ismael Botti ${ }^{1}$, Paulina Lira ${ }^{2}$, Jorge Martinez $^{2}$, Hagai Netzer ${ }^{3}$ \\ and Shai Kaspi ${ }^{3,4}$ \\ ${ }^{1}$ University of Nottingham, School of Physics \& Astronomy, Nottingham NG7, 2RD, U.K. \\ email: ppxib1@nottingham.ac.uk \\ ${ }^{2}$ Depto. de Astronomía, Universidad de Chile, Camino El Observatorio 1515, Santiago, Chile \\ email: plira@das.uchile.cl, jorgemarpa@ug.uchile.cl \\ ${ }^{3}$ School of Physics \& Astronomy and the Wise Observatory The Raymond and Beverly Sackler \\ Faculty of Exact Sciences Tel-Aviv University, Tel-Aviv 69978, Israel \\ email: netzer@wise.tau.ac.il \\ ${ }^{4}$ Physics Department, Technion, Haifa 32000, Israel \\ email: shai@wise.tau.ac.il
}

\begin{abstract}
We present an update of the monitoring campaign we have undertaken to probe the most massive black holes in powerful quasars at high redshift through the reverberation mapping technique. Once this campaign has finished, we will be able to directly measure broad line region (BLR) sizes of quasars at $z \sim 2-3$, improving dramatically the BLR size-luminosity relation, and therefore, black hole mass estimates based on this relationship. So far, we have identified a dozen highly variable sources suitable for future cross-correlation analysis and reverberation measurements.
\end{abstract}

Keywords. galaxies: high-redshift, quasars: general, quasars: emission lines

Reverberation mapping (RM) is the only tool to directly measure the broad-line region size and black hole mass in AGN in the distant universe. This technique assumes a BLR in virial equilibrium in the black hole potential well, and it relies on the ability to measure the time lag between the continuum variability from the accretion disk to that of the emission lines that arise from the BLR located further out the central engine (Peterson \& Horne 2005). RM has been successfully applied to low and intermediate luminosity AGN $\left(L \lesssim 10^{46} \mathrm{erg} \mathrm{s}^{-1}\right)$ in the local universe leading to the empirical relation between the BLR size and luminosity of the AGN (Kaspi et al. 2000, Bentz et al. 2011 and references therein), which has enabled black hole mass estimation through single-epoch observations (Vestergaard 2004). Kaspi et al. (2007) presented the first preliminary measurement of the BLR-size of a luminous quasar, however, the high luminosity end of the relation has not yet been probed statistically, and extrapolations (by even two orders of magnitude) are needed to estimate black hole masses in such systems.

Monitoring high luminosity AGN is a challenging task given the long time scales involved, dilated by the $(1+z)$ factor, and lower amplitude variability with respect to nearby AGN. In 2005, we started monitoring the continuum of a large sample of high luminosity quasars $\left(L \gtrsim 10^{46.5} \mathrm{erg} \mathrm{s}^{-1}\right)$ at high redshift $(z \sim 2.4-3.4)$ in the $R$-band. Spectroscopic follow up for each quasar was triggered after showing significant variability. So far, after $\sim 8$ years of continuum and spectroscopic monitoring, we have identified a dozen of objects with high variability which are suitable candidates for reverberation measurements. However, well populated light curves are needed to perform this method reliably. In figure 1 we show continuum and emission line light curves for some of our highly variable quasars. Some time lags can be noted between the continuum and emission line curves, but spectroscopic light curves are still too few populated for cross-correlation 

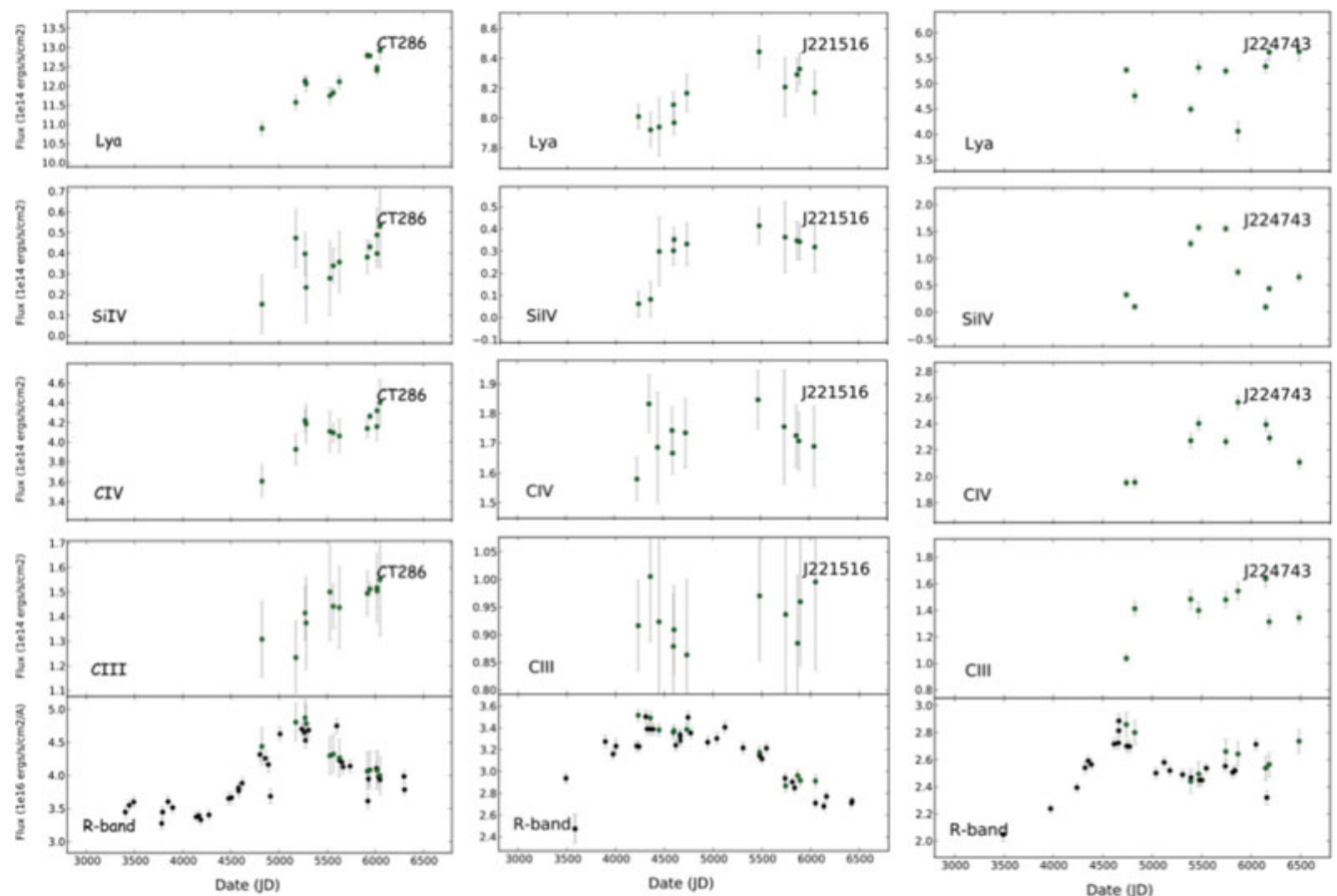

Figure 1. Continuum ( $R$-band) and UV emission line light curves for three of our highly variable quasars: CTQ 286 (left), J221516 (middle) and J224743 (right). High variability with breaks can be seen in the continuum, a key feature needed in cross-correlation analysis.

analysis. We expect to complete a denser emission line light curves in a few years time, and obtain the first reliable BLR-size and black hole mass estimates and probing the high end of the BLR size-luminosity relation with several objects for the first time, enabling reliable single epoch black hole mass estimates in AGN at all redshifts.

\section{References}

Bentz, M. C., Denney, K. D., Grier, C. J., et al. 2013, ApJ, 767, 149

Kaspi, S., Smith, P. S., Netzer, H., et al. 2000, ApJ, 533, 631

Kaspi, S., Brandt, W. N, Maoz, D., et al. 2007, ApJ, 659, 997

Peterson, B. M. \& Horne, K. 2005, Planets to cosmology : essential science in the final years of the Hubble Space Telescope, Space Telescope Science Institute symposium series, Vol. 18 (Cambridge University Press, 2006)

Vestergaard, M. 2002, ApJ, 571, 733 